\title{
Image Fusion Scheme based on Wavelet Transformation
}

\author{
Nisar Ahmed \\ Research Scholar, Mewar \\ University \\ Department of Electronics and \\ Communication \\ Rajasthan, INDIA
}

\author{
Ruhiat Sultana \\ Research Scholar, Rayalaseema \\ University \\ Department of Computer Science \\ and Engineering \\ Kurnool, INDIA
}

\author{
Syed Abdul Sattar, PhD \\ Professor \\ Department of Electronics and \\ Communication \\ Nawab Shah Alam Khan \\ Engineering College, Hyderabad, \\ INDIA
}

\begin{abstract}
The paper focuses on image fusion between multi-spectral images and panchromatic images using a gradient based wavelet analysis method with image processing traits. For highlighting the core features of source images, preprocessing is accomplished. A new gradient technique is developed based on wavelet transformation. As of gradient process, for a multi-spectral image, every pixel having 4 neighbors of each of them. The summation that we do matches those of the multi-spectral images that we have selected. Two major considerations for gradient are considered. One is for the pixel values in the multi-spectral images region and other is the gradient pixel values that are at the boundary. Once we have calculated these values, we transfer these values from the multi-spectral images onto the panchromatic image and then rest of the values outside the mask are kept as same. Since we are working on the RGB images, we copied the RGB channels directly. By calculating bigger gradient of the two images either in multi-spectral images or panchromatic images and then construct the final image using inverse wavelet transformation. The proposed method is experimented on satellite, medical and natural images. In all the cases, this method shown superiority with existing methods.
\end{abstract}

\section{General Terms}

Gradient pyramid, spatial frequency, Haar fusion, panchromatic image.

\section{Keywords}

Gradient, wavelet, fusion, Multi-spectral, pre-processing.

\section{INTRODUCTION}

In a multiband, few images represent only smooth variations i.e., weak features while few images have sharp and strong features: that depend on the substances exhibiting either stronger or weaker response for the given wavelength. We need to particularly preserve the weak features in the correct proportion during the fusion. Thus aim of multiband image fusion is to form a single image that merges possible features from all the source images. In the proposed method each pixel in the image band is weighted differently depending on its local details. The weight for any particular pixel in the image is computed based on its relative importance of the feature with respect to its neighborhood. Each pixel in the fused image is obtained with the normalized weighted average of all the pixels at the corresponding location in the band. Local details in the image are obtained by subtracting the $2 \mathrm{D}$ smoothed image from the original image. However, the smoothing filter should not blur the edges. Bilateral [1] and nonlocal means filter [2] is popular filters for preserving edges while smoothing. The fusion method based on the wavelet transformation has retained and inherited the main merits of tower-shaped transformation fusion method $[3,4,5]$. Simultaneously, because the orthogonal wavelet transformation has non-redundancy, the image data quantity after wavelet transformation will not increase. By using the direction of wavelet transformation, a better visual effect of fusion image will be able to obtain based on the vision characteristics that the human eye have different resolutions to high frequency component of different directions The image fusion realization requests to extract information (details) from each source image and obtains the effective demonstration in the final fusion image. According to the general theory of imagery processing, the image information (details) is concluded in the image high frequency component, therefore, the key point of image fusion research is to seek an appropriate processing method of fusing detailed information of source image respectively, that is, how will the information be fuse-processed effectively in the corresponding frequency band. According to the multi-resolution analysis theory, the source image after the wavelet transformation, the inner tube signal row, contains separately the image high frequency component of corresponding frequency directed in normal, horizontal and $45^{\circ}$ of the image, therefore, using the image tower-shaped structure after wavelets decomposing, to carry on fusion processing separately according to different transformation level and different frequency band, the information detail from different images can be fused effectively.

Carry on $\mathrm{N}$ wavelet transformation to the two-dimensional picture, and it will finally get some $(3 \mathrm{~N}+1)$ different frequency bands, which contains a $3 \mathrm{~N}$ high frequency bands and a low-frequency band. The image fusion program based on wavelet multi-dimensional transformation is shown as in Figure 2. Take two image fusions as an example, many image fusion methods may be analogized from this regarding. Suppose A and B are two primitive images, F is the image after fusion, its basic fusion steps are as follows:

Apart from medical and remote sensing, other fusion applications include classification [6], target identification [7] and object detection [8]. The areas in which multiband/multispectral image fusion find its applications include, geological surveying and environmental monitoring. Another popular category of fusion is in the area of remote sensing with multispectral (MS) and panchromatic (PAN) images. Multispectral images are usually characterized by its higher spectral but lower spatial resolution and vice-versa in the case of panchromatic images. There are quite a large 
number of papers that discuss on the fusion techniques for such images. In [9] authors have proposed the technique of combining multispectral and satellite aperture radar (SAR) images by intensity modulation. In [10] authors have carried out fusion based on component framework, whereas in [11] a specific image model has been formed for pan-sharpening approach. Fusion algorithm based on pyramid transforms such as Laplacian and Gaussian are constructed by filtering the images successively and then down sampling. Registered images are subjected to pyramid transform and then fused in the transformed domain. The final image is obtained by performing the inverse transform. The PCA transform decorrelates the inter-correlated multispectral bands into a new set of uncorrelated components. Only the first principal component which contains the most information of the MS image is substituted with PAN image. In recent years wavelet transforms have been successfully used. In [12] authors use wavelet packet transform to fuse Quickbird panchromatic and multispectral data fusion. But there are certain drawbacks of wavelet transform worth to be mentioned. With contourlet transform it is possible to avoid the mentioned drawbacks and hence in [13] authors have used contourlet based fusion for pan sharpening. Unlike the case, where features from high spatial resolution panchromatic sensor is injected into low spatial resolution multispectral bands, authors in [14] have taken into account the physical electromagnetic spectrum response of sensors during the fusion process. Authors showed that the obtained image would be closer to the image obtained by the ideal sensor than obtained by usual waveletbased fusion method. Authors in [15] have developed a fusion strategy based on synthetic variable ratio method to reproduce the spectral and spatial characteristics of multispectral and panchromatic images, respectively.

In this paper, we propose a novel fusion strategy by obtaining gradient weights from different images in a given multiband using wavelets. Gradient weights vary as the dominating features of the pixels in the individual image vary. Finally, a fused image is obtained with a gradient weighted sum of individual components.

The rest of the paper organized as follows: the sections 2 describe about proposed method, section 3 describes about pictorial and numerical results and discussions. Finally conclusions are derived in section 4.

\section{PROPOSED METHOD}

In a multi-spectral, few images represent only smooth variations i.e., weak features while few images have sharp and strong features: that depend on the substances exhibiting either stronger or weaker response for the given wavelength. We need to particularly preserve the weak features in the correct proportion during the fusion. Thus aim of multispectral image fusion, is to form a single image that merges strong features which are obtained from gradient of all the source images. In the proposed method each pixel in the image is gradient weighted differently depending on its local details. The gradient weight for any particular pixel in the image is computed based on its relative importance of the feature with respect to its neighborhood. Each pixel in the fused image is obtained with the normalized gradient weighted average of all the pixels at the corresponding location in the multi-spectral.

\subsection{Wavelet transformation}

The 1-Dwavelettransformofa1-Dsignal $f_{i}\left(x_{i}\right)$ [16]

$W(a, b)=\int_{-\infty}^{\infty} f_{i}\left(x_{i}\right) h_{a, b}{ }^{*}\left(x_{i}\right) d x_{i}$

Where * represents the complex conjugate.

A set of daughter wavelets $h_{a, b}\left(x_{i}\right)$ is generated from the mother wavelet by scale $\mathrm{a}$ and shift $\mathrm{b}$ operations

$$
h_{a, b}\left(x_{i}\right)=\frac{1}{\sqrt{a}}\left(\frac{x_{i-b}}{a}\right)
$$

Where $\sqrt{a}$ is a normalized factor.

The 2-Dwavelettransformofa1-Dsignal $f_{i}\left(x_{i}, y_{i}\right)$

$$
\begin{aligned}
& W\left(a_{1}, a_{2}, b_{1}, b_{2}\right) \\
& =\int_{-\infty}^{\infty} \int_{-\infty}^{\infty} f_{i}\left(x_{i}, y_{i}\right) h_{a_{1} a_{2} b_{1} b_{2}}{ }^{*}\left(x_{i}, y_{i}\right) d x_{i} d y_{i}
\end{aligned}
$$

A set of daughter wavelets $h_{a_{1} a_{2} b_{1} b_{2}\left(x_{i}, y_{i}\right)}$ is produced from the mother wavelet $h\left(x_{i}, y_{i}\right)$ by scales (a1 and a2) and shifts (b1 and b2) operations:

$$
h_{a_{1} a_{2} b_{1} b_{2}} b\left(x_{i}, y_{i}\right)=\frac{1}{\sqrt{a_{1} a_{2}}}\left(\frac{x_{i-b_{1}}}{a_{1}}, \frac{y_{i-b_{2}}}{a_{2}}\right)
$$

The different daughter wavelets are spatially multiplexed with different reference-beam directions [17].

\subsection{Basic steps of proposed method}

Consider two images, need to perform fusion operation, in general, two images are considered as input for fusion operation.

The basic fusion steps are as follows:

1. Apply wavelet transformation separately to each source image to establish various images of the wavelet tower-shaped transformation.

2. Apply fusion processing separately to each transformation level using different fusion operator, to obtain fusion wavelet pyramid

3. For each wavelet pyramid, apply gradient to obtain dominant features. The dominant features are very useful to reconstruct the fusion image from two images.

Apply the wavelet inverse transformation (i.e. to carry on image restructuring) to gradient result, obtain restructuring image namely for fusion image.

The proposed method block diagram is given in Fig. 1. 


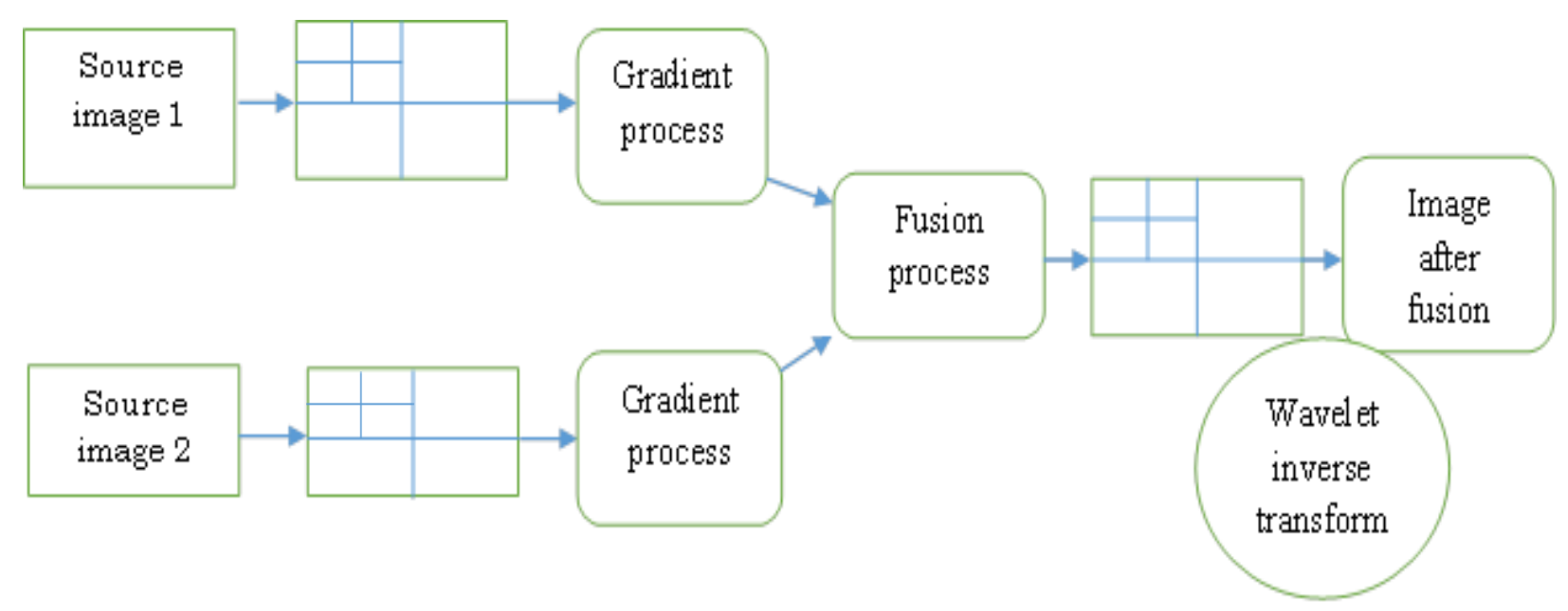

Fig 1: Block diagram of proposed method.

\subsection{Basic steps of proposed method}

Step 1: To highlight the dominant features of source and target images use pre-processing. By this, the source images are first padded so that they are the same size.

Step 2: Find multi spectra band images using single level 2-D discrete wavelet transformed to get $w_{L L}^{1}, w_{L H}^{2}, w_{H L}^{3}, w_{L L}^{4}$ subbands $\left[w_{L L}^{1}, w_{L H}^{2}, w_{H L}^{3}, w_{L L}^{4}\right]=D W T\left(W\left(x_{i}, y_{i}\right)\right.$.

Step 3: Calculating the gradient of each pixel of each band.

In a band image, every pixel has 4 neighbors. Two major considerations for gradient need to be made - one is for the pixel values in the source image region and other is the gradient pixel values that are at the boundary. Once we have calculated these values, we transfer these values from the source image onto the target or background image and then rest of the values outside the mask are kept as it is.

Step 4: Construct the fusion image using wavelet inverse transformation.

\section{RESULTS AND DISCUSSION}

We tested the proposed method on different images like satellite, medical and natural images. From these images, it is observed that each category of images have different set features and phenomena. The purpose of fusion is to form a single image by merging possible features from all the source images. Figure $2 \&$ Figure 3 Shows the fusion of images. The proposed method clearly identifies the image dominant features and gets success in merging them into single image. For evaluating the proposed algorithm quantitatively, we use statistical measures like average correlation, average Signal noise ratio (SNR) and entropy, mean of fusion image for qualitative measure. The correlation of first image and fusion image is $\mathrm{C} 1$ and correlation of second image and fusion image is $\mathrm{C} 2$ then considered average correlation is average of $\mathrm{C} 1$, C2. Similarly SNR ratio also. We compare results of multi spectral image fusion with (a) contourlet transform used in [18] and (c) bilateral approach suggested in [19] and tabulated in Table 1 .

The entropy is calculated using following equation

$$
\text { Entropy }=-\sum_{i} \sum_{j} f_{i j} \log f_{i j}
$$

This is a measure of randomness, having its highest value when the elements of $f$ are all equal.

The gradient of an image is given by the formula

$\nabla f=\left[\begin{array}{l}g_{x} \\ g_{y}\end{array}\right]=\left[\begin{array}{l}\frac{d f}{d x} \\ \frac{d f}{d y}\end{array}\right]$

Where:

$$
\begin{aligned}
& \frac{d f}{d x} \text { is the gradient in the } \mathrm{x} \text { direction } \\
& \frac{d f}{d y} \text { is the gradient in the y direction. }
\end{aligned}
$$

Table 1. Comparison of Counterlet Transform and Bilateral Approach.

\begin{tabular}{|l|l|l|l|l|}
\hline Methods & Average & Average & Entropy & Gradient \\
& Correlation & SNR(db) & & \\
\hline Contourlet & 85.65 & 17.36 & 4.6 & 4.3 \\
transform & & & & \\
\hline Bilateral & 90.12 & 19.68 & 5.4 & 4.9 \\
approach & & & & \\
\hline Proposed & 97.25 & 29.98 & 6.2 & 5.9 \\
method & & & & \\
\hline
\end{tabular}




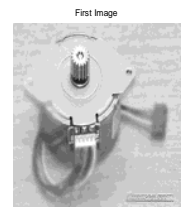

(a)

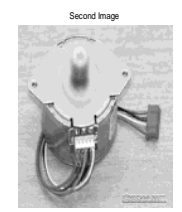

(b)

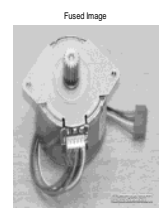

(c)
Fig 2: (a) First Image (b) Second Image \& (c) Fused Image

Correlation between first image and fused image $=0.977748$

Correlation between second image and fused image $=0.981530$

SNR between first image and fused image $=29.92 \mathrm{db}$

SNR between second image and fused image $=29.98 \mathrm{db}$

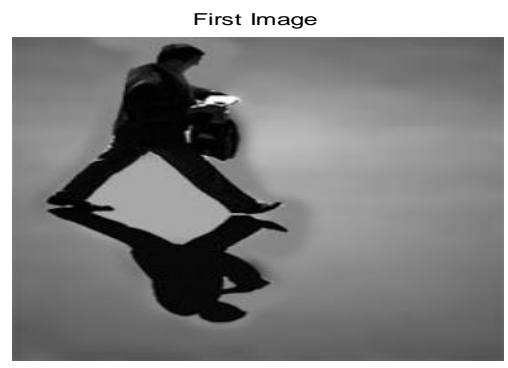

(a)

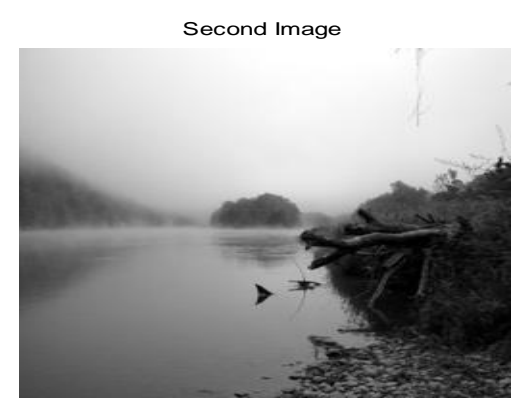

(b)

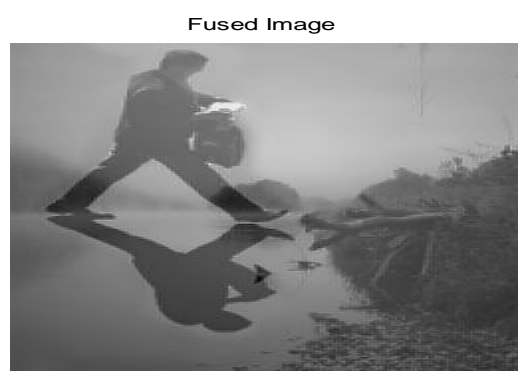

(c)

Fig 3: (a) First Image (b) Second Natural Image \& (c) Fused Image

Correlation between first image and fused image $=0.508583$

Correlation between second image and fused image $=0.843888$

SNR between first image and fused image $=14.30 \mathrm{db}$

SNR between second image and fused image $=14.30 \mathrm{db}$

\section{CONCLUSIONS}

In this paper, we have proposed a novel fusion strategy for different category of images. As the information across the images in the each category is not uniformly distributed, many traditional fusion methods based on averaging the images with equal weights fail. In the proposed method gradients computed on wavelet mutli- spectral images for fusion is based on the local information in each spectral image and hence are different for different images. Fused image is formed with the dominant average gradient of the pixels at the corresponding location. Reduction in the computational cost is achieved by a separable implementation of local gradient. We are able to achieve better results by exploiting all available information at all locations from all the images in the all wavelet spectral image. Results are tested qualitatively and quantitatively with a wide number of quality metrics and also compared with other fusion based approaches.

\section{REFERENCES}

[1] C. Tomasi and R. Manduchi, "Bilateral filtering for gray and color images," Proc. 6th Int. Conf. Computer vision. Washington, pp. 839-846, 1998.

[2] . A. Buades, B. Coll, and J. M. Morel, "A non-local algorithm for image denoising," in IEEE Computer Vision and Pattern Recogognition (CVPR), 2005, pp. 60-65.

[3] JIA Yong-hong, Li de-ren, SUN Jia-bing. Multidimensional remote sensing imagery data fusion. Remote sensing technology and application2000,15 (1):41-44

[4] LIU Xiao-long. Study on multi-resource remote sensing imagery information maintaining fusion technology. Journal of image and graphics, 2001, 6 (7):636-641.

[5] LI Jun, LIN Zong-jian. Remote sensing imagery data fusion method based on the characteristic Journal of image and graphics, 1997, 2 (2):103-107.

T. Westra, K. C. Mertens, and R. R. D. Wulf, "Waveletbased fusion of SPOT/VEGETATION and ENVISAT/ASAR wide swath data for wetland mapping," in SPOT/VEGETATION Users Conference, 2004, pp.24-26.

[6] Z. Korona and M. M. Kokar, "Multiresolution multisensory target identification," In J. D. Irwin (Ed.), The Industrial Electronics Handbook, vol. 12, pp. 1627-1632, 1997.

[7] Z. Zhang and R. S. Blum, "A region-based image fusion scheme for concealed weapon detection," in St Annual Conference on Information Sciences and Systems, 1997, pp. 168-173.

[8] L. Alparone, L. Facheris, S. Baronti, A. Garzelli, and F. Nencini, "Fusion of multispectral and sar images by intensity modulation," in Int. Conf. Inf. Fusion, Stockholm, Sweden, 2004, pp. 637-643.

[9] W. Dou, Y. Chen, X. Li, and Z. Sui, "A general framework for component substitution image fusion: An implementation using the fast image fusion method," Computational Geoscience, vol. 33, no. 2, pp. 219-228, 2007.

[10] M. Joshi, L. Bruzzone, and S. Chaudhuri, "A model-based approach to multiresolution fusion in 
remotely sensed images," IEEE Trans. Geosci. Remote Sens., vol. 44, no. 9, pp. 2549-2562, 2006.

[11] W. Zhang and J. Kang, "Quickbird panchromatic and multi-spectral image fusion using wavelet packet transform," Lecture Notes in Control and Information Sciences. Berlin, Germany: SpringerVerlag, vol. 344,pp. 976-981, 2006.

[12] V. Shah, N. Younan, and R. King, "An efficient pan-sharpening method via a combined adaptive pca approach and contourlets," IEEE Trans. Geosci. Remote Sens., vol. 46, pp. 1323-1335, 2008.

[13] X. Otazu, M. Gonzlez-Audcana, O. Fors, and J. Nnez, "Introduction of sensor spectral response into image fusion methods," IEEE Transactions on Geoscience and Remote Sensing, vol. 43, no. 10, 2005.
[14] Y. Zhang, "A new merging method and its spectral and spatial effects," International Journal of Remote Sensing, vol. 20, no. 10, pp. 2003-2014, 1999.

[15] Daubechies I.The wavelet transforms timefrequency localization and signal analysis. IEEE TransInfTheory 1990; 36:961-1005.

[16] Mendlovic D, Konforti N. Optical realization of the wavelet transform for two-dimensional objects. ApplOpt1993; 32:6542-6.

[17] A. Alejaily, I. Eirule, and M. Mangoud, "Fusion of remote sensing images using contourlet transform," in Intl. Conf. on Innovations and Advanced Technique in systems, Computing Sciences and Software Engineering, 2008, pp. 213-218.

[18] S. Raman and S. Chaudhuri, "Bilateral filter based compositing for variable exposure photography," in Eurographics Conf., Munich, Germany, 2009. 